\title{
Formale wissenschaftliche Kommunikation
}

Niels Taubert

\section{Gegenstand}

Eine zentrale Komponente der Wissenschaft ist die formale wissenschaftliche Kommunikation. "Wissenschaftlich" ist sie, weil sie sich an dem Code wahr/unwahr orientiert, indem sie Wahrheitsansprüche erhebt, unterstützt, kritisiert oder wiederlegt. Als „formal“ ist sie aus zwei Gründen zu bezeichnen: Zum einen besitzt die Kommunikation eine spezifische Form, nämlich die der Publikation, die sich durch Schriftlichkeit und Öffentlichkeit auszeichnet. Sie richtet sich an einen nicht spezifizierten, personell potentiell unbeschränkten Adressatenkreis und ihr geht ein förmlicher Akt der Veröffentlichung voraus. Zum anderen besitzt die formale wissenschaftliche Kommunikation eine besondere Form der Selbstreferenzialität. Wissenschaftliche Publikationen gehen u.a. aus Publikationen hervor (Schimank 2012, S. 116) und sie beziehen sich durch Zitate aufeinander. Die Besonderheit dieser Form von Selbstbezüglichkeit besteht in ihrem hohen Maß an Explizitheit, die es erlaubt, den Verweisen zu folgen. Die Verweisstruktur macht zudem deutlich, dass formale wissenschaftliche Kommunikation nicht etwa eine unstrukturierte Menge an Kommunikation ist, sondern ein integriertes System bildet. Dieses Merkmal wurde in der Literatur früh erkannt und die Gesamtheit der formalen wissenschaftlichen Kommunikation wird daher auch als formales wissenschaftliches Kommunikationssystem bezeichnet (s. exemplarisch Gravey und Griffith 1967, Whitley 1968).

Das formale wissenschaftliche Kommunikationssystem erfüllt vier Funktionen: Registrierung, Zertifizierung, Verbreitung und Archivierung (Andermann und Degkwitz 2004, S. 8; Taubert und Schön 2014, S. 7; Taubert und Weingart im Erscheinen):

Mit Registrierung ist die nachprüfbare Bestimmung des Zeitpunkts gemeint, zu dem ein Wahrheitsanspruch erhoben wird. Sie ist entscheidend für die Rekonstruktion des Wissensfortschritts in einem Feld und für die Zuordnung der Priorität von Wahrheitsansprüchen zu einer oder mehreren Personen.

- Zertifizierung meint die Anerkennung eines Beitrags als Teil eines gemeinsamen Wissensstands, häufig durch Begutachtung (Peer Review). Erst mit der Zertifizierung gilt ein Beitrag als von der wissenschaftlichen Gemeinschaft akzeptiert und in den Wissensbestand aufgenommen.

- $\quad$ Verbreitung bezeichnet die Herstellung von Reichweite innerhalb einer wissenschaftlichen Kommunikationsgemeinschaft. Die Referenz auf die Kommunikationsgemeinschaft spezifiziert die Öffentlichkeit von Publikationen. Der prinzipiell unbeschränkte Adressatenkreis wird dadurch eingeschränkt, dass das Verständnis ein hohes Maß an Fachkompetenz voraussetzt.

- $\quad$ Archivierung beschreibt die fortlaufende Sicherung eines Wissensbestands über längere Zeiträume, so dass an ihn zu einem späteren Zeitpunkt mit weiterer Forschung angeschlossen werden kann. Sie ist zudem Voraussetzung für die Beurteilung von Forschungsleistungen.

Vom formalen Kommunikationssystem zu unterscheiden sind die medientechnische Infrastruktur, die Voraussetzung für das formale Kommunikationssystems ist, sowie die Trägerorganisationen, die den Betrieb der Infrastruktur gewährleisten. 
Formale wissenschaftliche Kommunikation setzt eine leistungsfähige medientechnische Infrastruktur resp. Publikationsinfrastruktur voraus. Bestandteile sind sämtliche technische Einrichtungen, die eine oder mehrere der eben genannten Funktionen unterstützen. Die Form der Publikationsinfrastruktur unterliegt historischem Wandel. Nach der republic of letters, einer Phase des intensiven Austauschs zwischen Forschern und Gelehrten im 17. und 18. Jahrhundert per Brief fand eine Verbreitung der Kommunikation durch Medien statt, die allesamt der Druckerpresse entstammen. Dazu zählen gedruckte wissenschaftliche Journale ${ }^{1}$, Monographien, Conference-Proceedings und Sammelbände. Im Zuge der Digitalisierung sind elektronische Medien entstanden, die teils in ein Substitutions-, teils in ein Ergänzungsverhältnis zu gedruckten Medien getreten sind. Zu nennen sind hier elektronische Journale und Zeitschriftendatenbanken, die in zunehmendem Umfang gedruckte Zeitschriften ersetzen. Repositorien für Publikationen, die einen offenen Zugang zu an zugangsbeschränkten Orten veröffentlichten Publikationen ermöglichen, haben dagegen ergänzenden Charakter, da sie zwar die Verbreitung unterstützen, aber einen originären Publikationsort voraussetzen, an dem die Zertifizierung stattfindet. Die digitale Transformation betrifft nicht alle Medien gleichermaßen. So hat die elektronische Publikation sich zwar im Bereich der Journale bereits weitgehend durchsetzen können. Ungleich schwieriger ist die Situation bei Monographien - hier befinden sich elektronische Formate derzeit in der Erprobungsphase. Zur Publikationsinfrastruktur zählen darüber hinaus Einrichtungen, die die Begutachtung und technische Produktion von Publikationen unterstützen ${ }^{2}$ oder das Auffinden und die Rezeption von Publikationen erleichtern - etwa Bibliothekskataloge, Nachweissysteme, Fach- und Zitationsdatenbanken. Auch in diesem Bereich hat sich die Digitalisierung transformierend ausgewirkt. Neben gut eingeführten Einrichtungen sind digitale Plattformen wie ResearchGate oder Academia.edu hinzugekommen, die neben einer Bereitstellung von Publikationen mit ihrer Web 2.0-Funktionalität auch zu einer Vernetzung der wissenschaftlichen Gemeinschaft beitragen (vgl. Beiträge von Lüthje zu informeller Kommunikation und Schäfer zu Online-Kommunikation in diesem Band).

Die dauerhafte Verfügbarkeit von Infrastrukturen kann nur von Trägerorganisationen gewährleistet werden. Sie haben die Aufgabe, die Publikationsinfrastruktur zu warten und an sich wandelnde technische Bedingungen und Anforderungen der Nutzer anzupassen. Die Merkmale der Publikationsinfrastruktur sind eng mit den Aufgaben und dem Selbstverständnis der Trägerorganisationen verbunden. Während zu Zeiten der gedruckten Publikation die Aufgabe der Verlage in der Produktion, dem Inverkehrbringen und der Bewerbung von Veröffentlichungen lag und die Bibliotheken die Aufgabe wahrnahmen, durch die Organisation eines Verleihverkehrs die Reichweite von Publikationen zu erhöhen, sie zu archivieren und dauerhaft verfügbar zu machen, ist die Aufgabenteilung im Zuge des Medienwandels hin zur digitalen Publikation brüchig geworden. Verlage vertreiben Publikationen mithilfe von Online-Plattformen wie ScienceDirect (Elsevier) oder Springer Link (Springer) direkt an die Wissenschaftler und den Bibliotheken kommt hier vorrangig die Rolle des Beschaffers entsprechender Zugriffslizenzen zu. Auf der anderen Seite übernehmen Bibliotheken aber auch Aufgaben, die traditionell in die Domäne der Verlage fielen, indem sie beispielsweise Universitätsverlage betreiben oder Repositorien für die Archivierung und

$1 \quad$ Siehe zur Entstehung der Philosophical Transactions der Royal Society Zuckermann und Merton (1971). Instruktiv ist die historische Rekonstruktion, da sie den Zusammenhang zwischen der Entwicklung des Mediums und der Verfestigung der Registrierungs- und Zertifizierungsfunktion verdeutlicht.

2 Dies leisten zum Beispiel so genannte Online Editorial Management Systeme wie Open Journals System oder Editorial Manager. Siehe zu den Veränderungen, die solche Systeme im Begutachtungsprozess und bei der Zusammenarbeit zwischen Wissenschaftlern und Verlagen in den Sozialwissenschaften hervorrufen Taubert (2012). 
Zugänglichmachung von Publikationen bereithalten. Daher lässt sich von einer Verstärkung der Funktionskonkurrenz zwischen Verlagen und Bibliotheken sprechen. ${ }^{3}$

\section{Diversität der formalen Wissenschaftskommunikation}

Das formale Kommunikationssystem, Publikationsinfrastrukturen und spezifische Trägerorganisationen lassen sich in allen Bereichen der Wissenschaft ausmachen, gestalten sich aber sehr unterschiedlich aus. Ursache für die Diversität ist die ausgeprägte Binnendifferenzierung der Wissenschaft. Während sich diese bis vor kurzem noch als eine Differenzierung entlang von Disziplinen beschreiben ließ, ist die innere Struktur der Wissenschaft vor allem im Zuge der Orientierung an externen Erwartungen und der Herausbildung interdisziplinärer Forschungsgebiete unübersichtlicher geworden (für einen Überblick über disziplinäre Unterschiede und Besonderheiten vgl. Alexander von Humboldt-Stiftung 2009).

Bleibt man zunächst beim formalen Kommunikationssystem, dann zeigen sich erstens Unterschiede in der Dimension "Zeit" und hier insbesondere mit Blick auf die Publikationsgeschwindigkeit und die Dauer der Rezeption. Die Zeitdauer der Produktion von Forschungsergebnissen und der Veröffentlichung ist mit bestimmt durch das Ausmaß an Wettbewerb im Forschungsfeld, den Aufwand für die Niederschrift der Ergebnisse, von der Frequenz, mit der ein Publikationsmedium erscheint und letztlich auch durch das Verfahren, mit dem die Publikationswürdigkeit eines Manuskripts festgestellt wird. Die Rezeptionsdauer ${ }^{4}$ hängt primär von epistemischen Faktoren ab, wie dem Ausmaß, in dem auf der Grundlage einer gemeinsamen Theorie Wissensfortschritte erzielt werden und der daraus resultierenden Geschwindigkeit des Wissensfortschritts. Unterschiede in der Sachdimension sind zum Teil ebenfalls epistemischen Faktoren geschuldet. Während es die Naturwissenschaften meist mit Gegenständen zu tun haben, die weltweit für alle Wissenschaftlerinnen und Wissenschaftler des Fachs von Interesse sind, beschäftigen sich die Geistes- und Sozialwissenschaften häufig mit Gegenständen, die regional oder national geprägt und relevant sind. Daher variiert die Bedeutung der englischen Sprache je nachdem, ob ein Fach stark internationalisiert ist oder ein entlang von Sprachgrenzen fragmentiertes Kommunikationssystem aufweist (Hicks 1999; Nederhof 2006, S. 83f.). Unterschiede in der Sozialdimension beziehen sich auf den Autor oder die Gruppe von Autoren, denen die Publikation und die darin mitgeteilten Forschungsergebnisse zugeschrieben werden. Die Größe der Personengruppe schwankt stark (Nederhof 2006, S. 88), einerseits bedingt durch die Organisation der Forschung, andererseits durch unterschiedliche, fächer- oder forschungsfeldspezifische Kriterien, die Autorschaft konstituieren. Eine weitere Differenz in der Sozialdimension bezieht sich auf die Abgrenzung des Kommunikationssystems. Existiert ein Feld professioneller Tätigkeit, das abhängig ist von der Entwicklung des Wissenschaftsgebiets, sind die Grenzen der formalen Kommunikationssystems weniger scharf und es finden sich Publikationsformate, die einen Transfer leisten. Beispiele finden

3 Mit Verlagen und Bibliotheken sind die wichtigsten Trägerorganisationen benannt, die Aufzählung ist aber keineswegs vollständig. Komponenten der Publikationsinfrastruktur werden auch von wissenschaftlichen Fachgesellschaften (z.B. Journale), Informationsdienstleistern (z.B. die Zitationsdatenbanken des Web of Science, Thompson Reuters) und Forschungseinrichtungen (z.B. die Gesis als Betreiberin von Repositorien zur Publikation von Forschungsdaten) unterhalten.

4 Siehe zu den verschieden langen Zitationszeiträumen Glänzel und Schöpflin (1994, S. 58) und zum Alter referenzierter Literatur Glänzel und Schöpflin (1999, S. 41). 
sich in der medizinischen Forschung und der ärztlichen Praxis, den Ingenieurwissenschaften mit ihren Anwendungsfeldern sowie in der Rechtswissenschaft und der Rechtsprechung.

Auch die Publikationsinfrastruktur weist Fächer- oder forschungsgebietsspezifische Unterschiede auf. Variationen zeigen sich erstens bezüglich der Bedeutung, die den verschiedenen Typen von Publikationsmedien in einem Fach zukommt. In einigen Bereichen der Geistes- und Sozialwissenschaften wird die Monographie zur Mitteilung von Forschungsergebnissen nach wie vor genutzt (zur Bedeutung der Monographie in der Literaturwissenschaft bspw. Thompson 2002), während dem Journal-Artikel in den meisten Natur- und Lebenswissenschaften überragende Bedeutung zukommt (zur Bedeutung von Journal-Artikeln gegenüber anderen Publikationstypen Larivière et al. 2006, S. 1000 f., Bourke und Butler 1996, S. 475, Nederhof 2006, S. 84f.). In den Ingenieurwissenschaften sind auch Conference Proceedings relevant, die zum Teil ähnlich streng begutachtet werden wie Einreichungen in Journalen. In rasch fortschreitenden Forschungsgebieten sind dagegen Publikationsmedien anzutreffen, die Erkenntnisse in Form kurzer Mitteilungen und in oft erscheinenden Publikationsmedien - z.B. so genannte Letter-Journale (z.B. Physical Review Letters) - veröffentlichen, um eine schnelle Zirkulation der Ergebnisse zu erreichen. Zweitens differiert die Art von Struktur, die die Publikationsmedien eines Forschungsgebiets bilden. Bereits der Vergleich von Gebieten, in denen überwiegend in Journalen publiziert wird, offenbart große Unterschiede. An dem einen Extrem finden sich Fächer wie die Mathematik, die über eine thematisch breit gefächerte Journal-Landschaft verfügt und am anderen Extrem dagegen solche wie die Astronomie, deren Publikationsaktivität sich auf wenige, sehr große Journale konzentriert (de Boer 2009, S. 99). Daneben kann die Verteilung von Renommee der Publikationsmedien unterschiedliche Formen annehmen. Zudem schwankt auch die Bedeutung, die die gedruckte bzw. digitale Publikation in einem Fach hat. Diese Unterschiede sind u.a. für die gegenwärtige Diskussion um Open Access relevant, da der offene Zugang die digitale Publikation zur Voraussetzung hat.

Je nach Wissenschaftsgebiet variieren Art und Zusammensetzung der Trägerorganisationen. Richtet man seinen Blick zunächst auf die Wissenschaftsverlage, so scheint ein Korrespondenzverhältnis bzw. ein "lockeres Passungsverhältnis" (Schimank und Volkmann 2012, S. 170) zwischen den epistemischen Praktiken und der Struktur der Verlagslandschaft zu bestehen. Im Bereich von Science, Technology und Medicine (STM) sind wenige Großverlage wie Elsevier, Wiley oder Springer von zentraler Bedeutung, die mit mehreren tausend Mitarbeitern, auf der Grundlage einer internationalen Arbeitsteilung und unter Auslagerung wesentlicher Produktionsschritte ihr Publikationsgeschäft hochprofitabel betreiben. Dagegen sind in den Geistes- und Sozialwissenschaften vor allem kleine und mittelgroße Verlage anzutreffen, die nicht annähernd hohe Gewinne erwirtschaften. Die angesprochenen Unterschiede bezüglich des Anteils an Forschungsliteratur, die einen nationalen Sprachraum adressieren, sorgen dafür, dass die Märkte für Publikationen verschieden groß ausfallen. Mit der Größe von Wissenschaftsverlagen variieren auch das Ausmaß der Orientierung an ökonomischen Renditezielen (Schimank und Volkmann 2012; Volkmann et al. 2014) und der Einfluss eines Verlags auf die Gestaltung der weiteren Entwicklung der digitalen Publikation. Unterschiedliche Bedeutung kommt auch den Bibliotheken zu. In Fächern, in denen die gedruckte Publikation relevant ist, sind Bibliotheken in ihrer klassischen Rolle gefragt, indem sie Druckwerke erwerben, sammeln und einen Verleihverkehr organisieren. Findet der Zugriff dagegen überwiegend digital statt, liegt die primäre Aufgabe in der Herstellung des Zugangs durch den Erwerb von Zugriffslizenzen. 


\section{Forschungslinien}

Nach der Klärung des Gegenstands und der Beschreibung seiner Diversität sollen drei Forschungsfelder vorgestellt werden, die das formale Kommunikationssystem bereits seit längerer Zeit analysieren: Wissenschaftsforschung, Bibliometrie sowie Bibliotheks- und Informationswissenschaft. Ziel ist es dabei, die Breite der Zugänge illustrieren.

In der Wissenschaftsforschung standen bei der Beschäftigung mit dem formalen Kommunikationssystem lange vorrangig innerwissenschaftliche Strukturen und Prozesse im Mittelpunkt. Leitend war insbesondere die von der Mertonschen Wissenschaftssoziologie aufgeworfene Frage nach den „institutionellen Imperativen“ (Weingart 2003, S. 16), d.h. Normen, die die Erzeugung von sozial als wahr geltendem Wissen gewährleisten. Robert K. Merton fasst die normative Orientierung von Wissenschaftlern in einem Set institutionalisierter Normen, dem wissenschaftlichen Ethos (Merton 1942), zusammen, von dem er annimmt, es sei funktional für die Vermehrung von Wissen. Aus dieser Perspektive ist am formalen Kommunikationssystem vor allem interessant, ob die Norm ,Universalismus' gilt und die Prüfung von Wahrheitsansprüchen „unpersönlich“ (Weingart 2005, S. 69), d.h. ohne Ansehen von Kategorien wie Alter, Geschlecht und Herkunft der Autoren stattfindet. In dieser Tradition stehende Untersuchungen beziehen sich u.a. auf Entscheidungsprozesse über die Annahme eines Manuskripts zur Publikation (Crane 1967) und auf die Anerkennung von Forschungsleistungen im Zuge der Rezeption (Cole 1992, S. 158 ff.). Die in Teilen hier anschließende Peer Review-Forschung interessiert sich daneben für den Grad der Übereinstimmung von Gutachterurteilen, die dabei angelegten Gütekriterien und die Vorhersagevalidität der Gutachterempfehlungen (Hirschauer 2004, S. 64-73).

Im Kielwasser der "Laborstudien“, die sich für die Modalitäten der Wissensproduktion im Alltag naturwissenschaftlicher Labors interessieren (Merz 2006), hat sich die Perspektive der Wissenschaftsforschung verbreitert. Unter anderem sind zwei Phänomene in den Blick gekommen, die im strengen Sinne nicht zum formalen Kommunikationssystem zählen, aber für formale Wissenschaftskommunikation konstitutiv sind. Zum einen wird das Peer Review-Verfahren als komplexe Beobachtungskonstellation unter Beteiligung von Autoren, Gutachtern und Herausgebern (Hirschauer 2005) empirisch rekonstruiert. Dieser Perspektive nach wird das Verfahren nicht gleich einer dyadischen Prüfungssituation verstanden, in der zwei oder mehrere Statusrichter möglichst gleichgerichtete Urteile aussprechen, sondern als eine komplexe Beobachtungssituation, in der sich „unterschiedliche Urteile über wissenschaftliche Güte in ihrer eigenen Güte laufend selbst beobachten und kontrollieren" (ebd. 81). Zum anderen wird gefragt, welche Art von Transformation ein Wahrheitsanspruch von seinem Weg von der informellen Wissenschaftskommunikation des Labors hin zur formellen Wissenschaftskommunikation in Journalen durchläuft (Knorr-Cetina 2002, S. 175 ff; Myers 1990, vgl. auch Beitrag von Lüthje in diesem Band).

Die wissenschaftssoziologische Beschäftigung mit formaler Wissenschaftskommunikation ist nicht nur von der Theorieentwicklung und verschiedenen Methodologien geprägt, sondern auch von der Veränderung des Gegenstands. So hat beispielsweise das Entstehen großer Forschungskooperationen die Frage aufgeworfen, in welchem Sinn bei einer großen Anzahl von Autoren noch von einer Autorenverantwortung gesprochen werden kann und wie Forschungsleistungen angemessen zugerechnet werden können (Biagioli 2003, S. 255 ff.; Cronin 2005, S. 49 ff., Wray 2006, S. 508 ff.). Im Zuge Durchsetzung der digitalen Publikation lässt sich eine Ausweitung des Gegenstands beobachten. In den Blick genommen werden die bis dahin wenig 
beachteten Basistechnologien, auf denen das formale Kommunikationssystem basiert sowie die Rolle und Funktionsweise der Trägerorganisationen, wie Bibliotheken und Verlage (z.B. Hanekop und Wittke 2006, 2007; Schimank und Volkmann 2012; Volkmann et al 2014).

Eine zweite Forschungstradition, die Bibliometrie, zeichnet sich durch einen spezifischen methodischen Zugang zur formalen wissenschaftlichen Kommunikation aus. Sie ist letztlich nicht klar von der Wissenschaftsforschung abzugrenzen, da sich letztere häufig bibliometrischer Methoden bedient, wird hier aber als eigenständiger Zugang vorgestellt, da sie eine charakteristische Forschungsperspektive entwickelt hat und eine spezifische Art wissenschaftspolitischen Steuerungswissens bereitstellt. Typisch für diese Forschungstradition ist eine deskriptive Perspektive auf das wissenschaftliche Kommunikationssystem und die quantitative Untersuchung formaler Merkmale. Einer ihrer Ursprünge liegt in der Analyse des Wachstums des formalen Kommunikationssystems vor allem nach dem zweiten Weltkrieg, das sich u.a. mithilfe von Publikationszählungen gut darstellen lässt (de solla Price 1963). Von diesem Ausgangspunkt hat sich die Bibliometrie zu einem differenzierten Forschungsfeld entwickelt. Empirische Untersuchungen beschäftigen sich mit der Abbildung von Disziplinen und Forschungsfeldern durch Zitationsnetzwerke, den Unterschieden von Zitationsraten und Zitationsdauer in verschiedenen Forschungsfeldern, der Untersuchung von Zitationskartellen, der Karriere von Themen, der Entwicklung von Ko-Autorschaft und der Analyse (internationaler) Forschungskooperationen (vgl. die in Abschnitt 2 zitierte Literatur). Geprägt ist die Bibliometrie aber auch von ihrer Anwendung im Kontext der Leistungsmessung und Wissenschaftsevaluation. Publikationen und Zitationen werden genutzt, um Metriken zu errechnen, mit dem Ziel, die Leistungen von individuellen Forschern, Forschergruppen, Instituten, Universitäten oder ganzen Nationen zu vergleichen. Die Erfahrungen mit Wissenschaftsevaluationen haben gezeigt, dass die Messung von Forschungsleistungen nicht folgenlos ist, sondern das Publikationsgeschehen beeinflussen kann. Reaktanz tritt insbesondere dann auf, wenn die Leistungsmessung mit Anreizen - wie der Vergabe von Forschungsmitteln oder persönliche Bezüge - verknüpft wird. Dies veranlasst Wissenschaftler, ihre Publikationsstrategien an die Art der Leistungsmessung anpassen (zum Auftreten nicht intendierter Effekte überblicksweise Butler 2010, zum empirischen Nachweis von Anpassungsreaktionen, wie Strategien der Maximierung des Publikationsoutputs, Butler 2003, 2007).

Die Bibliotheks- und Informationswissenschaft ist ein praxisorientiertes Feld, dessen primärer Schwerpunkt weniger auf der Entwicklung von Theorie als in empirischen Fragen rund um die wissenschaftliche Informationsversorgung liegt. Ihr Gegenstandbereich schließt neben den Methoden des Informationszugangs, der Informationsorganisation und der Informationsgewinnung auch soft- und hardwaretechnische Voraussetzungen, Software-Ergonomie, rechtliche Regulationen, Bibliotheksorganisation und bibliometrische Methoden mit ein (Chua/Chang 2008, S. 2159). Weit über das Fach hinaus von Bedeutung ist die Diskussion um die Bibliothekskrise und darauf bezogene Reaktionen, die darauf abzielen, einen leserseitig offenen Zugang zu Publikation herzustellen (Open Access). In der bibliothekswissenschaftlichen Literatur wird seit Beginn der 1980er Jahre über ungewöhnlich hohe Preissteigerungen im Bereich der Subskriptionen für wissenschaftliche Fachzeitschriften berichtet (Panitch und Michalak 2005, Europäische Kommission 2006, S. 16; Kirchgässner 2008, S. 138; Boni 2010, S. 294). Als strukturelle Ursachen wird zum einen das bereits erwähnte Größenwachstum des formalen Kommunikationssystems unter der Bedingung stagnierender oder konstanter Bibliotheksetats diskutiert. Diese Lage zwingt Bibliotheken zu Abbestellung von Zeitschriften und sorgt für einen Rückgang der Einnahmen von Verlagen, die wiederum mit Preissteigerungen reagieren, um die Gewinne aus dem Zeitschriftengeschäft zu 
sichern (Tenopir und King 2000, S. 31f.). Zum anderen haben Untersuchungen zum Markt für wissenschaftliche Publikationen einige Faktoren herausgearbeitet, die der Preisbildung unter wettbewerblichen Bedingungen entgegenstehen. Dazu zählt die Nichtsubstituierbarkeit der Ware ,wissenschaftliche Zeitschrift', die starke Konzentration erheblicher Anteile an Zeitschriften in den Händen weniger Verlage und eine Nachfrage, die sich nicht unmittelbar durch Wissenschaftler, sondern stellvertretend durch Bibliotheken artikuliert (Brinzinger 2010, S. 334, Parks 2002, S. 324).

In Reaktion auf die Zeitschriftenkrise werden Anstrengungen unternommen, Veröffentlichungen auf digitalem Weg für den Leser frei zugänglich und nutzbar zu machen. Dies geschieht entweder am originären Publikationsort (Gold Open Access), oder durch die Ablage einer Kopie der an einem zugangsbeschränken Ort erschienenen Veröffentlichung auf einem eDoc-Server bzw. Repositorium (Green Open Access). Forschungsschwerpunkte der Bibliotheks- und Informationswissenschaft liegen in der Untersuchung des Nutzungsumfangs beider Typen von Open Access ${ }^{5}$ und in der Analyse möglicher Zitationsvorteile von Open Access-Publikationen. ${ }^{6}$

\section{Ausblick}

Formales wissenschaftliches Kommunizieren wandelt sich derzeit dramatisch. Teils ist dies durch die Digitalisierung angestoßen, teils durch die Leistungsmessung im Rahmen von Forschungsevaluation und teils durch die Orientierung der Großverlage an ökonomischen Renditezielen. Drei Beispiele sollen zeigen, dass dieser Wandlungsprozess keineswegs abgeschlossen ist.

Es finden sich Anzeichen, dass die bereits vor gut drei Dekaden entstandene Bibliothekskrise aktuell bleibt. Großverlage haben auf die wissenschaftspolitische Forderung nach Open Access reagiert und entsprechende Geschäftsmodelle entwickelt, indem sie die Finanzierung ihrer Journale in Teilen von Subskriptions- auf Publikationsgebühren umgestellt haben. Deren Höhe und die fortbestehende Angewiesenheit der Wissenschaft auf renommierte, Reputation versprechende Publikationsorte lassen vermuten, dass Open Access nicht zwangsläufig zu einer Entlastung der Bibliotheksetats führen muss, auch wenn sich die Finanzierungswege wandeln.

Bezüglich der Begutachtungsverfahren, mit denen die Publikationswürdigkeit von Manuskripten festgesellt werden, sind in Zukunft Veränderungen zu erwarten. Neu entstandene Möglichkeiten digitaler Medien, Mitglieder von Forschungsgemeinschaften zu vernetzen und über OnlinePlattformen am Begutachtungsprozess zu beteiligen, treffen zusammen mit der Kritik am traditionellen Peer Review. Dieses wird zum Teil als innovationsfeindlich, wenig zuverlässig und unwissenschaftlich wahrgenommen. Die Experimente mit neuen Formen wie dem Public Peer Review, Open Peer Review, Open Discussion sowie die radikale Idee, Beiträge zunächst zu veröffentlichen, um deren Relevanz im Anschluss im Zuge der Rezeption beurteilen zu lassen (,first publish, then filter"), deuten den Möglichkeitsraum an, der mit der Digitalisierung hier entsteht. Dabei wird es interessant sein zu sehen, welche Art von Lösung sich in welchen Fächern und Fachgebieten bewährt.

$5 \quad$ Siehe zum Nutzungsumfang von Green Open Access z.B. Swan und Brown 2005, S. 3, Antelmann 2006 , S. 89, Harnad et al. 2008, S. 38 und von Gold Open Access Morrison 2005, S. 4, Xia 2010, S.620.

$6 \quad$ Klassisch dazu Lawrence 2001, S. 521, daran anschliessend Antelman 2004, Harnad und Brody 2004, Hajjem et al. 2005 sowie zur kontroversen Diskussion Craig et al. 2007, Davis und Walters 2011). 
Letztlich kann auch davon ausgegangen werden, dass ein Bedarf nach der Bewertung von Forschungsleistungen fortbestehen wird und aus dem formalen Kommunikationssystem gewonnene Informationen dabei eine wesentliche Rolle spielen werden. Von Bedeutung sind hier derzeit die Grundeinheiten ,Publikation' und ,Zitation', doch diese Situation könnte sich bald ändern. Bei digitalen Publikationen fallen Informationen über deren Nutzung an, die statistisch ausgewertet werden können. Auf der Grundlage dieser Informationen werden bereits heute neue Indikatoren wie Klick- und Downloadzahlen sowie die Erwähnung von Publikationen in sozialen Netzwerken errechnet. Ein Beispiel dafür ist Altmetric (Aldie und Roe 2013).

Die drei Beispiele zeigen, dass formales wissenschaftliches Publizierens einem unabgeschlossenen Wandel unterliegt, bei dem aufeinander folgende Wellen (medientechnischer) Innovationen das Kommunikationssystem der Wissenschaft durchlaufen. Ein angemessenes Verständnis kann nur erzielt werden, wenn bei der Analyse dieser Entwicklungen wissenschaftliche Kommunikation, medientechnische Infrastruktur mit ihren technischen Eigenschaften sowie Trägerorganisationen berücksichtigt und die Querbezüge und Wechselwirkungen zwischen den drei Ebenen herausgearbeitet werden. 


\section{Recommended Reading}

Butler, Linda 2003. Modifying Publication Practices in Response to Funding Formulars. Research Evaluation 12 (1): 39-46.

Hirschauer, Stefan 2004. Peer Review Verfahren auf dem Prüfstand. Zum Soziologiedefizit der Wissenschaftsevaluation. Zeitschrift für Soziologie 33 (1): 62-83.

Nederhof, Anton J. 2006. Bibliometric monitoring of research performance in the Social Sciences and the Humanities: A review. Scientometrics 66 (1): 81-100.

Weingart, Peter 2005. Die Stunde der Wahrheit. Zum Verhältnis der Wissenschaft zu Politik, Wirtschaft und Medien in der Wissensgesellschaft. Weilerswist: Velbrück.

Zuckermann, Harriet und Robert K. Merton 1971. Patterns of evaluation in science: Institutionalization, structure and functions of the referee system. Minerva 9 (1): S. 66-100.

\section{Literatur}

Adie, Euan und William Roe 2013. Altmetric: enriching scholarly content with article-level discussion and metrics. Learned Publishing 26 (1): S. 11-17.

Alexander von Humboldt Stiftung (Hrsg.) 2009. Publikationsverhalten in unterschiedlichen Disziplinen. Beiträge zur Beurteilung von Forschungsleistungen. Bonn: Diskussionspapier der Alexander von Humboldt-Stiftung 12/2009. 2. erw. Aufl.

Andermann, Heike und Andreas Degkwitz 2004. Neue Ansätze in der wissenschaftlichen Informationsversorgung. Ein Überblick über Initiativen und Unternehmungen auf dem Gebiet des elektronischen Publizierens. Historical Social Research 29 (1): 6-55.

Antelman, Kristin 2004. Do Open-Access Articles Have a Greater Research Impact? College \& Research Libraries News 65: 372-382.

Antelman, Kristin 2006. Self-archiving practices and the influence of publisher policies in the social sciences. Learned Publishing 19 (2): 85-95.

Biagioli, Mario 2003. Rights or Rewards? Changing Frameworks of Scientific Authorship. In: Scientific Authorship. Credit and Intellectual Property in Science, hrsg. Mario Biagioli und Peter Gallison, 253-279. New York/London: Routledge.

Boni, Manfred 2010. Analoges Geld für digitale Zeilen: der Publikationsmarkt der Wissenschaft. Leviathan 38 (3): 293-312.

Bourke, P. und Linda Butler 1996. Publication Types, Citation Rates and Evaluation. In: Scientometrics 37 (3): S. 473-494.

Brinzinger, Klaus-Rainer 2010. Piraterie oder Allmende der Wissenschaften? Zum Streit um Open Access und der Rolle von Wissenschaft, Bibliotheken und Markt bei der Verbreitung von Forschungsergebnissen. Leviathan 38 (3): 331-346.

Butler, Linda 2003. Modifying Publication Practices in Response to Funding Formulars. Research Evaluation 12 (1): 39-46.

Butler, Linda 2007. Assessing University Research. A plea for a balanced approach. Science and Public Policy 34 (8): 565-574.

Butler, Linda 2010. Impacts of performance-based research funding systems: A review of the concerns and the evidence. In Performance-based Funding for Public Research in Tertiary 
Education Institutions: Workshop Proceedings, hrsg. OECD. OECD Publishing. http://dx.doi.org/10.1787/9789264094611-7-en.

Chua, Alton Y.K. und Christopher C. Yang 2008. The Shift Towards Multi-Disciplinarity in Information Science. Journal of the American Society for Information Sciences and Technology 59 (13): 21562170.

Cole, Stephen 1992. Making Science. Between Nature and Society. London: Harvard University Press.

Craig, Ian D., Andrew M. Plume, Marie E. McVeigh, James Pringle und Mayur Main 2007. Do Open Access Articles Have Greater Citation Impact. Journal of Informetrics 1 (3): 239-248.

Cronin, Blaise 2005. The Hand of Science. Academic Writing and its Rewards. Lanham et al: The Scarecrow Press.

Davis, Philip M. und William H. Walters 2011. The impact of free access to the scientific literature: a review of recent research. Journal of the Medical Library Association 99 (3): 208- 217.

Dickel, Sascha und Martina Franzen 2015. Wissenschaft im digitalen Wandel: Demokratisierung von Wissensproduktion und Wissensrezeption. WZB-Discussion Paper SP III 2015-601.

European Commission 2006. Study on the economic and technical evolution of the scientific publication markets in Europe. Final Report. Brussels: European Commission, DG Research. Online-Dokument: http://ec.europa.eu/research/science-society/pdf/scientific-publicationstudy_en.pdf.

Glänzeln, Wolfgang und Urs Schöpflin 1994. A stochastic model for the aging of scientific literature. Scientometrics 30 (1): 49-64.

Glänzeln, Wolfgang und Urs Schöpflin 1999. A bibliometric study of reference literature in the sciences and social sciences. Information Processing \& Management 35: 31-44.

Gravey, William D. und Belver C. Griffith 1967. Scientific communication as a Social System. Science 157 (3762): 1011-1016.

Hajjem, Chawki; Stevan Harnad und Yves Gingras 2005. Ten-Year Cross-Disciplinary Comparison of the Growth of Open Access and How it Increases Research Citation Impact. Bulletin of the IEEE Computer Society Technical Committee on Data Engineering. Online-Dokument: ftp://ftp.research.microsoft.com/pub/debull/A05dec/hajjem.pdf.

Hanekop, Heidemarie und Volker Wittke 2006: Das wissenschaftliche Journal und seine möglichen Alternativen: Veränderung der Wissenschaftskommunikation durch das Internet. In Internetökonomie der Medienbranche, hrsg. Svenja Hagenhoff, 201-233. Göttingen: Universitätsverlag Göttingen.

Hanekop, Heidemarie und Volker Wittke 2007: Der Einfluss des Internet auf die Re-Konfiguration des Systems wissenschaftlichen Publizierens. In Gesellschaft und die Macht der Technik. Sozioökonomischer und institutioneller Wandel durch Technisierung, hrsg. Ulrich Dolata und Reimund Werle, 201-220. Frankfurt/New York: Campus.

Harnad, Stevan und Tim Brody 2004. Comparing the Impact of Open Access (OA) vs. Non-OA Articles in the Same Journals. D-Lib Magazine 10 (6): Ohne Seitenzahl.

Harnad, Stevan, Tim Brody, François Vallières, Les Carr, Steve Hitchcock, Yves Gingras, Charles Oppenheim, Chawki Hajjem, Eberhard R. Hilf 2008. The Access/Impact Problem and the Green and Golden Roads to Open Access: An Update. Serials Review 34 (1): 36-40.

Hicks, Diana 1999. The difficulty of achieving full coverage of international social science literature and the bibliometric consequences. Scientometrics 44 (2): 193-215.

Hirschauer, Stefan 2004. Peer Review Verfahren auf dem Prüfstand. Zum Soziologiedefizit der Wissenschaftsevaluation. Zeitschrift für Soziologie 33 (1): 62-83. 
Hirschauer, Stefan 2005. Publizierte Fachurteile. Lektüre und Bewertungspraxis im Peer Review. Soziale Systeme 11 (1): 52-82.

Kirchgässner, Adalbert 2008: Zeitschriftenkonsortien. Angebotsausweitung auf Kosten der Flexibilität. In, Informationskonzepte für die Zukunft. ODOK '07 (Schriften der Vereinigung Österreichischer Bibliothekarinnen und Bibliothekare 5), hrsg. Eveline Pipp, 137-146. Graz-Feldkirch: Wolfgang Neugebauer Verlag GesmbH.

Knorr-Cetina, Karin 2002. Die Fabrikation von Erkenntnis. Zur Anthropologie der Naturwissenschaften. 2. erw. Neuaufl. Frankfurt: Suhrkamp.

Larivière, Vincent, Éric Archambault; Yves Gingras und Étienne Vignola-Gagné 2006. The Place of Serials in Referencing Practices: Comparing Natural Sciences and Engineering with Social Sciences and Humanities. Journal of the American Society for Information Science and Technology 57 (8): 997-1004.

Merton, Robert K. 1942. The normative Structure of Science. In The Sociology of Science. Theoretical and Empirical Investigations, hrsg. Robert K. Merton, 267-278. Chicago/London: The University of Chicago Press.

Merz, Martina 2006. The Topicality of the Difference Thesis. Revisiting Constructivism and the Laboratory. Science, Technology \& Innovation Studies. Special Issue 1: 11-24.

Morrison, Heather, G. 2005. The Dramatic Growth of Open Access: Implications and Opportunities for Resource Sharing. Online-Dokument: http://eprints.rclis.org/archive/00004558/02/dramatic.pdf

Myers, Greg 1990. Writing Biology: Text in the Social Construction of Scientific Knowledge. Madison: Universitäy of Wisconsin Press.

Nederhof, Anton J. 2006. Bibliometric monitoring of research performance in the Social Sciences and the Humanities: A review. Scientometrics 66 (1): 81-100.

Panitch, Judith M. und Sarah Michalak 2005. The Serials Crisis. A white Paper for the UNC-Chapel Hill Scholarly Communications Convocation, January, 2005. Online-Dokument: www.unc.edu/scholcomdig/whitepapers/panitch-michalak.doc

Parks, Robert P. 2002. The Faustian grip of academic publishing. Journal of Economic Methodology 9 (3): 317-335.

Schimank, Uwe 2012. Wissenschaft als gesellschaftliches Teilsystem. In Handbuch Wissenschaftssoziologie, hrsg. Sabine Maasen, 113-123. Wiesbaden: Springer.

Schimank, Uwe und Ute Volkmann 2012. Die Ware Wissenschaft: Die fremdreferentiell finalisierte wirtschaftliche Rationalität der Wissenschaftsverlage. In Wirtschaftliche Rationalität. Soziologische Perspektiven, hrsg. Anita Engels und Lisa Knoll, 165-183. Wiesbaden: Springer VS.

Swan, Alma und Sheridan Brown 2005. Open access self-archiving: An author study. UK: Key Perspectives limited. Als Online-Dokument. http://cogprints.org/4385/.

Taubert, Niels 2012. Online Editorial Management-Systeme und die Produktion wissenschaftlicher Fachzeitschriften. In: Leviathan 40 (2): 297-319.

Taubert, Niels und Kevin Schön 2014. Online-Konsultation „Publikationssystem”. Dokumentation und Auswertung. Persistent Identifier: urn:nbn:de:kobv:b4-opus-26293.

Taubert, Niels und Peter Weingart (im Erscheinen). Herausforderungen an das wissenschaftliche Kommunikationssystem. In Wissenschaftliches Publizieren: Wandel, Herausforderungen, Perspektiven, hrsg. Peter Weingart und Niels Taubert. Berlin: Walter de Gruyter.

Tenopir, Carol und Donald King 2000. Towards Electronic Journals. Washington: SLA Publishing.

Thompson, Jennifer Wolfe 2002. The death of the scholarly monograph in the humanities? Citation patterns in literary scholarship. Libri 52: 121-136. 
Volkmann, Ute, Uwe Schimank und Markus Rost 2014. Two Worlds of Academic Publishing: Chemistry and German Sociology in Comparison. Minerva 52 (2): 187-212.

Weingart, Peter 2003. Wissenschaftssoziologie. Bielefeld: transcript.

Weingart, Peter 2005. Die Stunde der Wahrheit. Zum Verhältnis der Wissenschaft zu Politik, Wirtschaft und Medien in der Wissensgesellschaft. Weilerswist: Velbrück.

Whitley, Richard D. 1968. The formal communication system of science: A study of the organisation of British social science journals. The Sociological Review 16 (1): 162-179

Wray, K. Brad 2006. Scientific authorship in the age of collaborative research. Studies in History and Philosophy of Science 37: 505-514.

Xia, Jingfeng 2010. A Longitudinal Study of Scholars Attitudes and Behaviors Towards Open-Access Journal Publishing. Journal of the American Society for Information Science and Technology 61 (3): 615-624.

Zuckermann, Harriet und Robert K. Merton 1971. Patterns of evaluation in science: Institutionalization, structure and functions of the referee system, Minerva 9 (1): 66-100. 


\begin{abstract}
Der Beitrag beschäftigt sich mit der formalen wissenschaftlichen Kommunikation, also denjenigen innerwissenschaftlichen Austauschprozessen, mit der Wahrheitsansprüche mitgeteilt, geprüft und diskutiert werden. Um einen für die Erfassung rezenter Wandlungsprozesse angemessenen Rahmen bereitzuhalten, wird der Gegenstand als Trias konzipiert, indem zwischen der formalen wissenschaftlichen Kommunikation, der Publikationsinfrastruktur und den Trägerorganisationen unterschieden wird. Merkmal der Kommunikation ist ihre Öffentlichkeit und die Adressierung einer wissenschaftlichen Gemeinschaft. Sie erfüllt die vier Funktionen der Registrierung, Zertifizierung, Verbreitung und Archivierung von Forschungsergebnissen und realisiert sich fach- und forschungsfeldspezifisch in sehr unterschiedlicher Weise. Vor allem mit der Wissenschaftssoziologie, Bibliometrie und Bibliotheks- und Informationswissenschaft finden sich Forschungstraditionen, die formale wissenschaftliche Kommunikation untersuchen. Der Beitrag stellt diese Traditionen überblicksartig vor und schließt mit einem Ausblick auf Wandlungsprozesse, die vor allem von der Digitalisierung geprägt werden.
\end{abstract}

\title{
Keywords
}

Formale Wissenschaftskommunikation, Publikationsinfrastruktur, Open Access, Wissenschaftsverlage, Bibliotheken, Wissenschaftssoziologie, Bibliometrie, Bibliotheks- und Informationswissenschaft, Bibliothekskrise, Peer Review

\section{Angaben zum Autor}

Niels Taubert, Jg. 1972, Dr. phil., Studium der Soziologie in Hamburg und Bielefeld, promovierte 2006 zur Open Source-Softwareentwicklung und arbeitet derzeit an einer Habilitationsschrift zu Open Access in Astronomie und Mathematik. Arbeitsschwerpunkte: Wissenschaftssoziologie, Techniksoziologie, Mediensoziologie, wissenschaftliches Publikationssystem, Open Access. eMail-Adresse: post@niels-taubert.de 